\title{
CONSUMO DE ALCOOL ENTRE UNIVERSITÁRIOS DO INTERIOR DO NORDESTE BRASILEIRO
}

\author{
ALCOHOL CONSUMPTION AMONG BRAZILIAN NORTHEASTERN COLLEGE \\ STUDENTS OF INTERIOR
}

\author{
Janieiry Lima de Araújo ${ }^{a^{*}}$, Sheila Maria da Conceição Costa ${ }^{b^{*}}$, Priscila Mikaelly Fernandes Mon- \\ teiro $^{\mathrm{b}^{*}}$, Ana Raquel Figueiredo Rego ${ }^{\mathrm{d}^{* *}}$, Ellany Gurgel Cosme do Nascimento ${ }^{\mathrm{e}^{* * *}}$ \\ janieiry@hotmail.comª sheilacosta2@hotmail.com ${ }^{\mathrm{b}}$, priscilamikaelly_pdf@hotmail.com ${ }^{\mathrm{b}}$, anaraquelfr@hotmail.com ${ }^{\mathrm{d}}$, \\ ellanygurgel@hotmail.com ${ }^{e}$
}

Universidade Estadual do Rio Grande do Norte - UERN*, Secretaria Municipal de Saúde de Pombal/PB ${ }^{* *}$, Secretaria Municipal de Saúde de São Miguel/RN**

Data de recebimento do artigo: 14/01/2019

Data de aceite do artigo: 18/02/2019

\section{RESUMO}

Objetivo: Descrever e analisar o consumo de álcool entre universitários. Método: Estudo descritivo e quantitativo. A coleta feita por aplicação de questionário com 369 estudantes da Universidade do Estado do Rio Grande do Norte. Os dados foram tabulados e analisados com auxílio do Statistical Package for Social Sciences. Resultados: Os participantes apresentam idade média de 22,11 anos, predominância do sexo feminino (63,68\%), estado civil solteiro (84,82\%), renda familiar de 1 a 3 salários mínimos (70,46\%). No padrão de consumo de álcool observou-se que 70,18\% ingeriram bebida alcoólica, o principal motivo foi por pura diversão (32,04\%), a média de início foi aos 16 anos. $40,1 \%$ afirmaram terem ficado de 'porre'. A interferência na saúde mais citada foi de problemas para alimentar-se (34,74\%). Dos que ingeriram álcool, 2,7\% sofreram acidentes sobre efeito do álcool e 1,54\% referiram a ocorrência de situações de violência. Observou-se que houve uma redução de consumo no último ano $(52,3 \%)$ e na última semana $(23,3 \%)$. Conclusão: A problemática do consumo de álcool, está presente desde antes da entrada na universidade e continua, posteriormente, tornando os universitários vulneráveis a esse agravo. Há, portanto, necessidade de intervenções de promoção a saúde sobre o uso de álcool e suas consequências.

Palavras-Chave: Consumo de bebidas alcoólicas; estudantes. saúde pública; bebidas alcóolicas; vulnerabilidade em saúde

\section{ABSTRACT}

Objective: Describe and analyze the alcohol consumption among college students. Method: A descriptive and quantitative study. The collect was made using a questionnaire with 369 students from the University of Rio Grande do Norte. The data were tabulated and analyzed using the Statistical Package for Social Sciences. Results: Participants have an average age of 22.11 years, female predominance (63.68\%), unmarried $(84.82 \%)$, family income $1-3$ minimum wages $(70.46 \%)$. In the pattern of alcohol consumption it was observed that $70.18 \%$ used alcohol, the main reason was for fun $(32.04 \%)$, the beginning average was 16 years. $40.1 \%$ said they had been 'drunk. The most frequently cited health complication was problems to eat (34.74\%). 2.7\% of those who drank suffered accidents on influence of alcohol and $1.54 \%$ reported the occurrence of violent situations. It was observed a reduction in the consumption of last year (52.3\%) and last week (23.3\%). Conclusion: The issue of alcohol consumption is present since before eturn the university and continues thereafter, making the college students vulnerable to this grievance. Therefore, health promotion interventions on alcohol use and its consequences are needed.

Keywords: Alcohol drinking; students; public health; alcoholic beverages; health vulnerability 


\section{Introdução}

A problemática do consumo de álcool tornou-se uma prioridade para a saúde pública mundial e nacional. O Relatório Mundial sobre Álcool e Saúde da Organização Mundial da Saúde, coloca que o consumo de álcool é o terceiro maior fator de risco no mundo para doenças e incapacidades, se tornando ainda maior nos países de renda média ${ }^{(1)}$.

Este documento traz ainda o álcool como causa direta de 60 doenças e lesões, complementar de outras 200 enfermidades e $4 \%$ da totalidade de mortes ocorridas no mundo, valor que supera a mortalidade por HIV/AIDS, a violência ou a tuberculose. Vale ressaltar a face social do problema do uso de álcool que envolve a violência, negligência infantil e abusos, além do absenteísmo no trabalho ${ }^{(1)}$.

No Brasil, o consumo de álcool vem sendo estimulado entre os jovens pela mídia e pela cultura, associado à ausência de políticas públicas reguladoras do comércio e acesso por parte desta população, o que torna o acesso à bebida alcoólica fácil e com baixo custo. Tais fatores motivam $65 \%$ dos adolescentes a experimentar a substância alguma vez na vida e, são responsáveis, pelo consumo de álcool por $69 \%$ dos adultos ${ }^{(2)}$.

Reconhecidos os investimentos científicos e de responsabilidade sociais imbuídos aos universitários brasileiros, as pesquisas têm-se voltado para este público de modo a determinar a prevalência do consumo de álcool, observando a compreensão que nossos estudantes têm sobre o uso nocivo da substância ${ }^{(3)}$.

$O$ ingresso na universidade traz à vida do jovem inúmeras mudanças, colocando-o em situação de instabilidade e consequente vulnerabilidade. Novas relações são construídas, existe uma constante necessidade de independência, além de responsabilização pelos seus próprios atos. Essas características da nova vida podem implicar no uso de bebidas alcóolicas como uma forma de escapar da rotina cansativa e estressante de estudos ${ }^{(4)}$.

Destarte, o ambiente da universidade tem influência direta sobre o início ou permanência do consumo desta substância ${ }^{(5)}$. De tal constatação surge o interesse da comunidade científica em estar pesquisando a inclusão do problema do consumo de álcool nas universidades brasileiras.
Sendo assim, o presente estudo objetivou descrever e analisar o consumo de álcool entre os estudantes universitários dos cursos de graduação de um Campus da Universidade do Estado do Rio Grande do Norte (UERN), localizado no município de Pau dos Ferros/RN.

\section{Metodologia}

Estudo descritivo, com abordagem quantitativa e amostragem probabilística estratificada. Foi realizado com universitários dos nove cursos de graduação existentes no Campus Avançado Pro$\mathrm{f}^{\mathrm{a}}$. Maria Elisa Albuquerque Maia (CAMEAM) da UERN, situado na cidade de Pau dos Ferros/RN.

Este trabalho representa um recorte de uma pesquisa de iniciação científica intitulada de "Uso de Álcool e Drogas por Universitários do Campus Avançado Prof ${ }^{\text {a }}$. Maria Elisa de Albuquerque Maia - UERN", e os resultados aqui discutidos referem-se às variáveis sobre o uso de álcool pelos universitários dos cursos de graduação em Enfermagem, Educação Física, Letras - Língua Portuguesa, Letras - Língua Espanhola, Letras - Língua Inglesa, Administração, Ciências Econômicas, Geografia e Pedagogia, os quais formam o universo desta pesquisa. Considerou-se, neste estudo, que cada curso de graduação do CAMEAM/UERN representa um subgrupo/estrato.

Para a realização do cálculo amostral, consideramos um Intervalo de Confiança de 95\%, assumindo um erro amostral de 5\%. Ainda adotamos como porcentagem de prevalência do evento a que foi encontrada em um estudo realizado na Universidade de São Paulo em 1997, que corresponde a 40\% (6). A amostra necessária para relevância do estudo foi 369 sujeitos. Para compensar as possíveis perdas no momento da coleta de dados, foi acrescida a amostra um valor de $20 \%$ a mais de participantes da pesquisa, assegurando assim, que fosse colhido um número significante de questionários. Ao final, atingimos a amostra ( $\mathrm{n}=369$ alunos).

O instrumento utilizado foi um questionário autoaplicável adaptado de uma pesquisa realizada por Galduróz et al (2004) e Stempliuk (2004) ${ }^{(7,8)}$, com questões objetivas e subjetivas em relação ao consumo de álcool e outras drogas (exceto tabaco). 
Para a seleção dos participantes da pesquisa, foi utilizado o software Microsoft Excel, através de uma ferramenta que faz a seleção aleatória das pessoas, que são representadas por números. Os critérios de seleção dos participantes foram: ser aluno regularmente matriculado no CAMEAM/ UERN e ter idade superior a 18 anos; e os de exclusão foram: ausência do aluno em sala da aula, impossibilidade de responder devido à deficiência motora manual permanente ou temporária, deficiência visual que o impeça de ler o questionário, alunos em processo de movimentação interna e/ ou trancamento.

A pesquisa foi submetida ao CEP/UERN eaprovada, em 15/05/12 sob a CAAE 03199812.8.0000.5294, e todas as etapas foram realizadas nos meses de novembro de 2012 a janeiro de 2013.

Os questionários coletados foram organizados por curso e por período, e foram tabulados com auxílio do software de computador Statistical $\mathrm{Pa}$ ckage for the Social Sciences (SPSS). A análise foi feita por estatística descritiva.

\section{Resultados e discussões}

\section{- Perfil dos Universitários}

Os universitários participantes (369 graduandos) revelaram as seguintes características: faixa etária de 18 a 35 anos (sendo a média da idade de 22,11 anos), predominância do sexo feminino $(63,68 \%)$ e estado civil solteiro(a) $(84,82 \%)$.

Quanto ao perfil socioeconômico em sua maioria residem com os pais $(68,02 \%)$, não têm filhos $(89,7 \%)$, são católicos $(70,18 \%)$, não praticantes da religião escolhida $(66,98 \%)$ e não possuem atividades remuneradas (57,72\%). Ao nos reportarmos para a escolaridade dos familiares, referiram que o pai e a mãe tinham ensino fundamental incompleto, sendo as porcentagens $48,23 \%$ e $40,1 \%$, respectivamente. A renda familiar declarada por $70,46 \%$ dos jovens foi entre 1 a 3 salários mínimos.

\section{- Perfil do Consumo de Álcool}

O uso de álcool vem sendo atualmente uma problemática caracterizada pelos índices crescentes de consumo no âmbito mundial e nacional. Quase 2 bilhões de pessoas no mundo fazem uso de álcool ${ }^{(3)}$. Observou-se neste estudo que $70,18 \%$ dos universitários já teriam feito uso de álcool.

Segundo I Levantamento Nacional sobre os padrões de consumo de álcool na população brasileira, 75\% já ingeriu algum tipo de bebida alcoólica ${ }^{(9)}$. Diante deste alto consumo de álcool, os universitários merecem destaque, pois possuem um comportamento vulnerável quanto ao uso, como demonstra estudo realizado em uma universidade pública de São Paulo, onde $84,7 \%$ dos universitários pesquisados utilizavam álcool ${ }^{(10)}$, um realizado em duas faculdades de Alagoas que teve a prevalência de uso de álcool de $90,4 \%$ pelos estudantes ${ }^{(11)}$, e outro feito em uma faculdade de direito no Maranhão onde o consumo de álcool entre os jovens estudantes chegou a $91 \%{ }^{(12)}$.

Percebe-se que os achados de consumo de álcool entre os universitários da UERN, estão um pouco abaixo do apresentado no I Levantamento Nacional Sobre o Uso de Álcool, Tabaco e outras Drogas entre Universitários das 27 Capitais Brasileiras ${ }^{(3)}$, em que $86,2 \%$ dos universitários brasileiros referiram já ter feito o uso de álcool em algum momento da vida. Já em outro estudo, 75\% da amostra relatou fazer uso da bebida, pelo menos, uma vez ao mês ${ }^{(4)}$.

Na Tabela 1, são elencados os principais motivos que levaram ao consumo da bebida, onde $32,04 \%$ tiveram a diversão como o motivo mais influenciador do uso.

Tabela 1 - Caracterização dos universitários do CAMEAM/UERN relacionada aos motivos que influenciaram o consumo de álcool.

\begin{tabular}{llc}
\hline Qual motivo de utilizar álcool? & $\boldsymbol{F}$ & $\%$ \\
\hline Diversão & 83 & 32,04 \\
Curiosidade & 48 & 18,53 \\
Influencia & 36 & 13,89
\end{tabular}




\begin{tabular}{lcc} 
Festas & 30 & 11,58 \\
Entrosamento & 25 & 9,65 \\
Vontade/Gostar & 24 & 9,26 \\
Outros & 41 & 15,78 \\
\hline
\end{tabular}

Fonte: Dados da pesquisa "Uso de álcool e drogas por universitários do Campus Avançado Profa. Maria Elisa de Albuquerque Maia - UERN", 2013.

A respeito do uso de álcool, 68,56\% foi antes do ingresso na universidade. $\mathrm{O}$ dado vai de encontro a outros estudos, onde a grande maioria $(92,7 \%)$ relatou este hábito como antecedendo ao ingresso na universidade ${ }^{(4)}$.

Os períodos letivos citados como de início do uso de álcool foram do primeiro ao nono período, sendo o primeiro período $(71,66 \%)$ o de maior predominância. Além disso, 15,55\% não informaram.

Apesar da relação álcool e jovens ser arriscada e trazer ônus para os jovens e para a sociedade, o consumo precoce de bebida alcoólica ocorre muito frequentemente pela maioria das pessoas. Esta problemática vem sendo silenciada e negligenciada pelo poder público e pela sociedade que consente tal comportamento no momento em que o encara como comum ou normal ${ }^{(13)}$.

Os universitários costumam fazer uso de álcool em ambientes festivos. Um estudo confirma esta informação e defende o fato de que nas festas, geralmente, existe uma oferta ilimitada de bebidas, além de estímulos do próprio ambiente, como a música alta e outras pessoas bebendo. Espaços de festas e bares possuem, inconscientemente, normas que le- vam o indivíduo ao uso abusivo de bebidas ${ }^{(14)}$.

Deste modo, alguns fatores podem estar relacionados ao uso do álcool, como as grandes propagandas instigadoras do consumo desta droga que frequentemente passa na mídia (televisão, internet, revistas, outros), em conjunto com a liberação legal de seu uso, bem como a facilidade de acesso, como discute o estudo com universitários de Maceió, onde 27,2\% relataram ter consumido devido o anúncio da mídia ${ }^{(11)}$.

Em relação a idade daqueles do uso de álcool pela primeira vez, as mais citadas foram 16 $(19,76 \%)$ e 15 anos (16,2\%), sendo a média de idade foi de 16 anos. A média de idade do uso de álcool corrobora ao evidenciado no I Levantamento Nacional sobre o Uso de Álcool, Tabaco e outras Drogas entre Universitários das 27 Capitais Brasileiras ${ }^{(3)}$, onde $54 \%$ dos entrevistados já haviam experimentado alguma bebida alcoólica antes dos 16 anos de idade.

Dos motivos alegados como justificativa para o uso de álcool após ingressar na universidade, a mais citada foi também "diversão" $(32,77 \%)$, conforme disposto na Tabela 2.

Tabela 2 - Caracterização dos universitários do CAMEAM/UERN relacionada aos motivos que influenciaram o consumo de álcool após a entrada na universidade.

\begin{tabular}{lcc}
\hline Motivos para o uso de álcool após entrar na universidade & F & \% \\
\hline Diversão & 59 & 32,77 \\
Festas/Comemorações & 42 & 23,33 \\
Entrosamento & 17 & 9,44 \\
Vontade/Gostar & 15 & 8,33 \\
Influencia & 14 & 7,77 \\
Relaxar & 13 & 7,22 \\
Costume & 13 & 7,22 \\
Outros & 23 & 12,72 \\
\hline
\end{tabular}

Fonte: Dados da pesquisa "Uso de álcool e drogas por universitários do Campus Avançado Profa. Maria Elisa de Albuquerque Maia - UERN”, 2013. 
Daqueles que referiram já terem feito uso de álcool, 40,1\% afirmaram já ter ficado de "porre". O achado encontra-se abaixo da descrição em outro estudo, onde 73,60\% dos participantes relataram já ter vivenciado um episódio de intoxicação aguda ("tomando um porre") pelo menos uma vez na vida ${ }^{(15)}$.

É importante salientar que junto ao consumo de álcool vem também as suas consequências físicas, psíquicas e sociais a vida dos sujeitos, já que dados mostram que cerca de 30 milhões de brasileiros já sofreram algum tipo de problema devido o uso de álcool, além de que esta substância psicoativa foi responsável por $3,2 \%$ de todas as mortes mundiais e por $4 \%$ de anos de vida útil perdidos ${ }^{(16)}$.

Em relação às interferências ocorridas no organismo e na vida dos participantes em decorrência do uso de álcool, destaca-se as consequências na alimentação $(34,74 \%)$ e no sono $(24,32 \%)$, conforme segue na Tabela 3.

Tabela 3 - Caracterização dos universitários do CAMEAM/UERN relacionada às interferências após o uso do álcool.

\begin{tabular}{lcc}
\hline Interferências após o uso do álcool & F & \% \\
\hline Não interfere & 149 & 57,52 \\
Alimentação & 90 & 34,74 \\
Sono & 63 & 24,32 \\
Atividade física ou esportiva & 36 & 13,89 \\
Desempenho nos estudos & 28 & 10,81 \\
Relações sociais afetivas & 21 & 8,1 \\
No trabalho & 13 & 5,01 \\
Outros & 17 & 6,53 \\
\hline
\end{tabular}

Fonte: Dados da pesquisa "Uso de álcool e drogas por universitários do Campus Avançado Profa. Maria Elisa de Albuquerque Maia - UERN", 2013.

O uso abusivo de álcool pode ser causador de acidentes de trânsito em consequência do efeito depressor desta droga, bem como seu uso crônico pode ocasionar dependência e afetar negativamente vários órgãos do corpo, como fígado, rins, pâncreas, estômago, entre outros, entrando assim como fator de risco para diversas patologias ${ }^{(4,16-18)}$.

Em relação ao sono, este é considerado um fator de risco para a ocorrência de acidentes de trânsito, algo comprovado por estudo toxicológico realizado pelo Instituto de Medicina Forense em São Paulo que mostrou que 50,6\% destes acidentes ocorreram em pessoas alcoolizadas ${ }^{(13)}$, o que remete a uma suscetibilidade para ocorrência destes acidentes no público alvo da pesquisa. Dos achados, 2,7\% referiram ter sofrido algum tipo de acidente sob o efeito do álcool, o que foi inferior a outros estudos, como o que avaliou o consumo de álcool em vítimas de acidentes, que encontrou valor de $16,8 \%$ de incidência ${ }^{(19)}$.

Já referente à violência, apenas $1,54 \%$ dos que consumiram bebidas alcoólicas referiram ter vivenciado algum episódio enquanto estavam alcooliza- dos. A ocorrência de violência após uso do álcool foi baixa, quando comparada com estudo que indicou a ocorrência de brigas de estudantes universitários sob efeito do álcool em 16,5\% dos casos (5). Quando se faz a busca relacionada aos casos de violência nos atendimentos de urgência no Brasil, $37,9 \%$ dos sujeitos envolvidos são suspeitos de estarem utilizando álcool no momento do ato ${ }^{(19)}$.

Além disso, a dificuldade de desempenho nos estudos teve incidência relevante $(10,81 \%$ dos universitários), situação discutida em outros estudos, tendo em vista que muitos universitários começam a ter um mal desempenho acadêmico em decorrência do uso de álcool, como consequência do baixo rendimento em sala de aula, além do déficit de memória e dificuldade de aprendizagem causada ${ }^{(17,20)}$. Estas problemáticas podem ser visualizadas em pesquisa realizada na Escola de Enfermagem de Ribeirão Preto da Universidade de São Paulo, onde se verificou que $71,2 \%$ dos estudantes dormem em aula após ter ido à festa na noite anterior e ter ingerido álcool na ocasião, sendo que $50 \%$ chegam atrasados em sala de aula ${ }^{(17)}$. 
Dentro desta problemática de ótica acadêmica, verificou-se que os participantes deste estudo afirmaram consumir bebidas alcoólicas durante o desempenho das atividades na universidade $(6,5 \%)$, e irem à faculdade sob efeito do álcool (11,11\%), o que provavelmente potencializa as consequências.
Relacionando o uso de álcool com o período de tempo ao qual foi utilizado, obtiveram-se as informações da Tabela 4 , onde se observa que o seu consumo vem reduzindo de um ano atrás $(52,3 \%)$ comparado à última semana $(23,3 \%)$.

Tabela 4 - Caracterização dos universitários do CAMEAM/UERN relacionada ao tempo de uso do álcool.

\begin{tabular}{ccccccccc}
\hline \multirow{2}{*}{$\begin{array}{c}\text { Uso de Álcool } \\
\text { F }\end{array}$} & \multicolumn{2}{c}{ Ultima Semana } & \multicolumn{2}{c}{ Ultimos 30 dias } & \multicolumn{2}{c}{ Ultimos 6 meses } & \multicolumn{2}{c}{ Ultimos 12 meses } \\
\cline { 2 - 9 } & $\mathbf{\%}$ & $\boldsymbol{F}$ & $\mathbf{\%}$ & $\boldsymbol{f}$ & $\mathbf{\%}$ & $\boldsymbol{f}$ & $\mathbf{\%}$ \\
\hline Não & 222 & 60,16 & 170 & 46,07 & 125 & 33,87 & 105 & 28,45 \\
Sim & 86 & 23,3 & 133 & 36,04 & 174 & 47,15 & 193 & 52,30 \\
Não faço uso & 56 & 15,17 & 61 & 16,53 & 63 & 17,07 & 66 & 17,88 \\
Não informou & 5 & 1,35 & 5 & 1,35 & 7 & 1,89 & 5 & 1,35
\end{tabular}

Fonte: Dados da pesquisa "Uso de álcool e drogas por universitários do Campus Avançado Profa. Maria Elisa de Albuquerque Maia - UERN”, 2013.

Quando se remete à periodicidade, as informações se assemelham aos resultado do I Levantamento Nacional sobre o Uso de Álcool, Tabaco e outras Drogas entre Universitários das 27 Capitais Brasileiras, onde o consumo reduziu de $72 \%$ nos últimos 12 meses para $60,5 \%$ nos últimos 30 dias, o que demonstra a conformidade do resultado do trabalho em questão com a realidade do país ${ }^{(3)}$.

Observando o tipo de bebida alcoólica consumida, a mais utilizada foi a cerveja $(43,08 \%)$, seguida por cachaça (5,14\%). Quanto as companhias que eles têm ao ingerirem álcool, a mais citada foi com os amigos $(58,68 \%)$, seguido de familiares $(18,91 \%)$ e sozinhos $(8,49 \%)$. E o lugar que mais costumam tomar bebidas é em festas e baladas (52,89\%), seguida de em bares (19,69\%) e em casa $(12,35 \%)$.

Assim, os universitários podem ser elencados como um público vulnerável e com o processo saúde-doença desgastado, sendo um dos agravantes o uso de álcool ${ }^{(13)}$.

\section{Conclusão}

A pesquisa evidencia o início precoce de consumo do álcool, mesmo antes de entrar na universidade, vinculados aos momentos festivos e na companhia de amigos. Destaca-se que os achados do estudo realizado numa cidade do interior do Brasil se assemelha aos encontrados nas grandes Instituições de Ensino Superior (IES) espalhadas pelo país e no I Levantamento Nacional sobre o Uso de Álcool, Tabaco e outras Drogas entre Universitários das 27 Capitais Brasileiras, deste modo afirmando a disseminação e popularização na aceitação de normalidade do consumo de álcool entre jovens.

Espera-se que os achados aqui dispostos sejam fonte de reconhecimento da problemática, e que contribua para a construção de projetos de intervenções, pelo poder público e pelos coordenadores das universidades, no intuito de agir para minimizar as consequências do consumo de álcool pelos universitários.

\section{Referências}

1. World Health Organization. Global status report on alcohol and health. WHO Genebra: Library Cataloguing-in-Publication Data; 2011. ISBN 9789241564151.

2. Silva CJ, Laranjeira R. Diagnóstico e tratamento do uso nocivo e dependência do álcool. Diagnóstico e tratamento em clínica médica - Psiquiatria. 2006 Fev 14;47-64.

3. Brasil; Presidência da República; Secretaria Nacional de Políticas sobre Drogas. I Levantamento Nacional sobre o Uso de Àlcool, Tabaco, e outras Drogas entre Universitários de 27 Capitais Brasileiras/Secretaria Nacional de Políticas sobre Drogas; GREA/IPQ-HC/FMUSP; organizadores Arthur Guerra de Andrade, Paulina do Carmo Arruda Vieira Duarte, Lúcio Garcia de Oliveira. - Brasília: SENAD, 2010. 284 p. 
4. Ramis TR, Mielke GI, Habeyche EC, Oliz MM, Azevedo MR, Hallal PC. Tabagismo e consumo de álcool em estudantes universitários: prevalência e fatores associados. Rev Bras Epidemiol. 2012 Jun;15(2):376-85.

5. Wagner GA, Andrade AG. Uso de álcool, tabaco e outras drogas entre estudantes universitários brasileiros. Rev Psiquiatr Clín. 2008 Fev 20;35 Suppl 1:48-54.

6. Andrade AG, Queiroz S, Villaboim RCM, Casar CLG, Alves MCGP, Bassit AZ, et al. Uso de álcool e drogas entre alunos de graduação da Universidade de São Paulo. Rev ABPAPAL. 1997;19(2):53-9.

7. Carlini EA, Galduróz JCF, Noto AR, Fonseca AM; Centro Brasileiro de Informações sobre Drogas Psicotrópicas (CEBRID). V levantamento nacional sobre o consumo de drogas psicotrópicas entre estudantes do ensino fundamental e médio da rede pública de ensino nas 27 capitais brasileiras - 2004. CEBRID/UNIFESP. 2004;11-381.

8. Stempliuk VA. Uso de drogas entre alunos da Universidade de São Paulo: 1996 versus 2001 [tese]. São Paulo: Faculdade de Medicina da Universidade de São Paulo; 2004. 149 p.

9. Laranjeira R, Pinsky I, Zaleski M, Caetano R; Secretaria Nacional Antidrogas (SENAD). I Levantamento nacional sobre os padrões de consumo de álcool na população brasileira. CEBRID/UNIFESP. 2007;8-76.

10. Silva LVER, Malbergier A, Stempliuk VA, Andrade AG. Fatores associados ao consumo de álcool e drogas entre estudantes universitários. Rev Saude Publica. 2006 Abr;40(2):280-8.

11. Pedrosa AAS, Camacho LAB, Passos SRL, Oliveira RVC. Consumo de álcool entre estudantes universitários. Cad Saude Publica. 2011 Aug;27(8):1611-21.

12. Carvalho DA, Gomes RIB, Sousa VEC, Sardinha AHL, Filho MRC. Hábitos alcoólicos entre universitários de uma instituição pública. Cienc Cuid Saude. 2011 Jul/ Set;10(3):571-77.

13. Vieira DL, Ribeiro M, Romano M, Laranjeira RR. Álcool e adolescentes: estudo para implementar políticas municipais. Rev Saude Publica. 2007 Mar 29;41(3):396-403.

14. Filho NH, Teixeira MAP. Motivos para beber e situações de consumo de bebidas alcoólicas: um estudo exploratório. Mudanças - Psicologia da Saúde. 2012 Jan/Dez;20(1-2):1-6.

15. Oliveira M, Soibelmann M, Rigoni M. Estudo de crenças e expectativas acerca do álcool em estudantes universitários. Int J Clin Saúde Psychol. 2007;7(2):421-33.

16. Andrade AG, Oliveira LG. Principais consequências em longo prazo relacionadas ao consumo moderado de álcool. In: Andrade AG, Anthony JC, Silveira CM. Álcool e suas consequências: uma abordagem multiconceitual. Barueri: Minha Editora; 2009. p. 37-66.
17. Pillon SC, Corradi-Webster CM. Teste de identificação de problemas relacionados ao uso de álcool entre estudantes universitários. R Enferm UERJ. 2006 Jul/Set;14(3):325-32.

18. Gomes BMR, Alves JGB, Nascimento LC. Consumo de álcool entre estudantes de escolas públicas da Região Metropolitana do Recife, Pernambuco, Brasil. Cad Saude Publica. 2010 Apr;26(4):706-12.

19. Mascarenhas MDM, Malta DC, Silva MMA, Carvalho CG, Monteiro RA, Neto OLM. Consumo de álcool entre vítimas de acidentes e violências atendidas em serviços de emergência no Brasil, 2006 e 2007. Cien Saude Colet. 2009 Nov/Dec;14(5):1789-96.

20. Pechansky F, Szobot CM, Scivoletto S. Uso de álcool entre adolescentes: conceitos, características epidemiológicas e fatores etiopatogênicos. Rev Bras Psiquiatr. 2004 May;26 Suppl 1:14-17.

Como citar este artigo:

Costa SMC, Monteiro PMF, Araújo JL, Rego ARF, Nascimento EGC. Consumo de Álcool entre Universitários do Interior do Nordeste Brasileiro. Rev. Aten. Saúde. 2019;17(59):88-94. 